\title{
Mit Katzenbildern fing es an
}

Der Begriff der „Artificial Intelligence“ (AI) wurde das erste Mal von Computerspezialisten auf der Dartmouth Conference 1956 vorgestellt. Computerprogramme, die Dame spielten, waren eines der frühesten Anwendungsbeispiel für AI. Seitdem wird prophezeit, dass AI der Schlüssel zur neuen und besseren Zukunft der Menschheit ist. Wirklich?

„Deep Learning” mit künstlichen neuronalen Netzen ist eine besonders effiziente Methode des permanenten maschinellen Lernens auf der Basis der statistischen Analyse großer Datenmengen (Big Data) und die bedeutendste Zukunftstechnologie innerhalb der AI. Das Heraussuchen von Katzenbildern auf YouTube war eine der ersten erfolgreichen Demonstrationen von Deep Learning. Inzwischen hat Google DeepMind's AlphaGo den Großmeister in dem Brettspiel „Go”, Lee Se-Dol, besiegt.

\section{Vom Helferlein zum Entscheider?}

Lange wurde es nicht für möglich gehalten, dass eine Maschine den Menschen beim "Go" schlagen kann, weil es fast unendlich viele Spielvarianten gibt. Aber eben nur fast unendlich. Heutzutage ist die Bilderkennung durch trainierte Maschinen bei einigen Aufgaben schon besser als der Mensch. Sehr erfolgreich agiert etwa das Deep Learning Convolutional Neural Network $(\mathrm{CNN})$. In einer aktuellen Studie konnte das CNN bei der Beurteilung dermatoskopischer Aufnahmen gut- und bösartige Hautläsionen besser differenzieren als selbst eine internationale Gruppe erfahrener Dermatologen.

Mancher Arzt mag ganz zufrieden damit sein, dass es eine intelligente diagnostische Hilfe gibt, die ihm die Arbeit ab- nimmt. Aber es wird auch Kollegen geben, die sich fragen, ob dies nicht der erste Schritt ist, mit dem die Maschine den Arzt Schritt für Schritt ganz klammheimlich als Entscheider in der Behandlung seiner Patienten ablöst.

Vielleicht ist das gar nicht so schlecht. Wenn der Arzt von zeitraubenden Aufgaben wie der Befundung von dermatoskopischen Aufnahmen entlastet wird, dann hat er mehr Zeit für das Gespräch mit dem Patienten. Mehr Empathie und weniger Reparaturannahmestelle - das ist es auch, was sich der Patient wünscht. Das ist leider nicht die Kernkompetenz so mancher fachlich kompetenter Ärzte.

Wir sollten nicht darauf warten, bis die $\mathrm{AI}$ auch diese Aufgabe übernimmt. Big Data hat bereits eine Sprachsoftware entwickelt, bei der man telefonisch einen Tisch im Restaurant bestellen kann, ohne dass der Gegenüber am anderen Ende der Telefonleitung bemerkt, dass er mit einer Maschine spricht. Und computergestützte automatisierte Programme („Bots") werden nicht nur für die politische Meinungsmache in sozialen Netzen entwickelt, sondern auch für medizinische Anwendungen. "Woebot" ist beispielsweise ein freundlicher Kummer-Chatbot für depressive Patienten. Psychologen der Universität Stanford haben ihn programmiert. Wäre dies nicht auch etwas für die psychoonkologische Versorgung?

AI wird dazu beitragen, dass der Beruf des Arztes in Zukunft anders definiert werden wird. Wir wissen nur noch nicht, wie. Mit Katzenbildern fing es an.

Dr. Alexander Kretzschmar, München

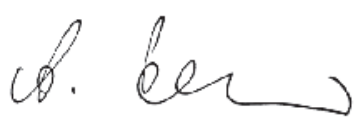

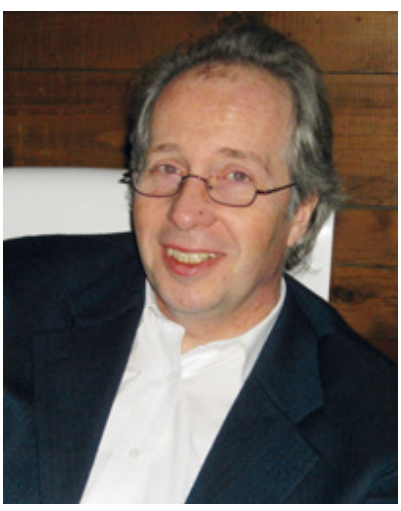

Dr. Alexander Kretzschmar 